\title{
Quenching of $1^{+}$excitations in the double giant resonance
}

\author{
C.A. Bertulani ${ }^{a, b}$, V.Yu. Ponomarev ${ }^{a, c}$ and V.V. Voronov ${ }^{c}$ \\ ${ }^{a}$ Institut für Kernphysik II, GSI, \\ Planckstr. 1, D-64291 Darmstadt, Germany \\ ${ }^{b}$ Permanent address: Instituto de Física, \\ Universidade Federal do Rio de Janeiro \\ 21945-970 Rio de Janeiro, RJ, Brazil, e-mail: bertu@if.ufrj.br \\ ${ }^{c}$ Permanent address: Bogoliubov Laboratory of Theoretical \\ Physics, Joint Institute for Nuclear Research, 141980, Dubna, Russia
}

\begin{abstract}
The electromagnetic excitation of the two-phonon isovector giant dipole resonance in relativistic projectiles incident on heavy targets can be proceed via several intermediate $1^{-}$one-phonon giant resonance states. In two step electric dipole transitions the population of $0^{+}, 1^{+}$, and $2^{+}$two-phonon states are possible. We calculate the amplitude distribution of $1^{-}$excitations with an RPA formalism, and use it to calculate the electromagnetic excitation of two-phonon states in second order perturbation theory and coupled-channels. We show that a conspiracy between angular momentum coupling and the strength of the electromagnetic fields suppresses contributions of $1^{+}$states to the total cross sections.
\end{abstract}

Keywords: Two-Phonon Giant Dipole Resonance, Electromagnetic Excitation, Quenching PACS: 24.30.Cz, 25.70.De, 25.75+r 
The electromagnetic excitation of two-phonon states of giant dipole resonances in heavyion collisions at relativistic energies has been studied in several experiments [1 [5]. In LAND measurements [1].2.5] these cross sections were extracted from total cross sections by separating a contribution coming from excitation of single dipole and quadrupole resonances. The observed cross sections are large, on the order of several hundred of millibarn. However, the first experimental results for the double giant dipole resonance (DGDR) excitation were found to be enhanced by factors of $2-3$ for ${ }^{136} \mathrm{Xe}[\mathrm{I}]$ and ${ }^{197} \mathrm{Au}[2]$ as compared to any theoretical calculations available. Although for the later measurements in ${ }^{208} \mathrm{~Pb}$ [5] the experiment-theory correspondence is much better, theoretical calculations still underpredict the DGDR cross section by about $30 \%$. Another part of the problem with double resonances is related to the experimental position and width of these new resonances and how they correspond to a harmonic picture of nuclear excitation. For more details of experimental studies of this problem we refer to reviews [6].

There are only a few theoretical papers written on this subject. In the first group the GDR is treated phenomenologically as a single state and the DGDR is either a sum of these two GDR [7] or a sum of two "GDR" phenomenologically mixed with "GDR" ${ }^{N}$ " states [8]. These papers deal mainly with the problem related to the excitation cross section of the DGDR. The position and width of the DGDR is a subject for the second group [9 13] where the GDR is considered microscopically. In ref. 10 a fine structure of the GDR was calculated by coupling of RPA $1^{-}$states to two-phonon configurations and the DGDR was treated as two independent excitations of the single GDR. In other papers eigen wavefunctions of the DGDR were used and two-phonon DGDR states were coupled either (a) between themselves in ref. [9], or (b) to $1 \mathrm{p} 1 \mathrm{~h}$ and $2 \mathrm{p} 2 \mathrm{~h}$ configurations within second RPA approach in ref. [1], or (c) to three phonon configurations in ref. [12], or (d) to all of them in ref. [13]. Somewhat aside from these two groups is the paper [14] where general properties of the DGDR are treated by means of a sum rule approach.

In the present paper we investigate the contribution of non-natural parity $1^{+}$two-phonon states to the total electromagnetic excitation cross sections. The $\left[1^{-} \otimes 1^{-}\right]_{1^{+}}$component of the DGDR was never considered in previous microscopic studies. They could, in principle, be responsible for the missing part of theoretical evaluations of the DGDR excitation cross sections. In phenomenological approaches describing the single GDR as one collective state, this component of the DGDR is forbidden by symmetry properties. Taking into account the Landau damping this collective state splits into a set of different $1_{i}^{-}$states distributed over an energy interval, where $i$ stands for the order number of each state. Again, the diagonal components $\left[1_{i}^{-} \otimes 1_{i}^{-}\right]_{1^{+}}$are forbidden by the same symmetry properties but nondiagonal 
ones $\left[1_{i}^{-} \otimes 1_{i^{\prime}}^{-}\right]_{1^{+}}$, a priori, may be excited in two-step process bringing some "extra strength" in the DGDR region. Consequently, the role of these nondiagonal components depends on how strong is the Landau damping.

To take into account the Landau damping effect we have performed an RPA calculation for $J^{\pi}=1^{-}$states in ${ }^{208} \mathrm{~Pb}$ making use the quasiparticle-phonon model [15]. The model Hamiltonian includes an average field for protons and neutrons, treated by a Woods-Saxon potential, and a residual interaction in a separable form. For open shell nuclei it also has a term corresponding to monopole pairing. For our RPA calculation we have used the single particle spectrum and parameters of residual interaction from ref. [16]. This calculation provides us with the spectrum $E_{i}$ of one-phonon $1^{-}$states and reduced matrix elements $<1_{i}^{-}\|E 1\|$ g.s. $>$ of their electromagnetic excitation from the ground state. We produce two-phonon DGDR states with quantum numbers $J^{\pi}=0^{+}, 1^{+}$and $2^{+}$by coupling onephonon RPA states with the wave function $\mid 1_{i}^{-}>_{m}$, to each other. The index $m$ stands for different magnetic substates. The wave function of the two-phonon states has the form:

$$
\left|\left[1_{i}^{-} \otimes 1_{i}^{-}\right]_{J^{\pi}=0^{+}, 2^{+}}>_{M}=\frac{1}{\sqrt{2}} \sum_{m, m^{\prime}}\left(1 m 1 m^{\prime} \mid J M\right)\right| 1_{i}^{-}>_{m} \mid 1_{i}^{-}>_{m^{\prime}},
$$

for two-phonon states made of two identical phonons while for other DGDR states it is:

$$
\left|\left[1_{i}^{-} \otimes 1_{i^{\prime}}^{-}\right]_{J^{\pi}=0^{+}, 1^{+}, 2^{+}}>_{M}=\sum_{m, m^{\prime}}\left(1 m 1 m^{\prime} \mid J M\right)\right| 1_{i}^{-}>_{m} \mid 1_{i^{\prime}}^{-}>_{m^{\prime}},
$$

In the present calculation we do not include the interaction between DGDR states, of eqs. (0.1-0.2), and we do not couple them to states with different than two number of phonons as was done in ref. [13]. Thus, our two-phonon states $\mid\left[1_{i}^{-} \otimes 1_{i^{\prime}}^{-}\right]_{J^{\pi}}>_{M}$ have excitation energy equal to the sum of one-phonon energies $E_{i}+E_{i^{\prime}}$ and are degenerated for different values of the total spin $J^{\pi}$ and its projection $M$. The reduced matrix element $<\left[1_{i^{\prime}}^{-} \otimes 1_{i}^{-}\right]_{J^{\pi}}\|E 1\| 1_{i}^{-}>$ of electromagnetic excitation of two-phonon states, eqs. (0.1-0.2), from the one-phonon state $\mid 1_{i}^{-}>_{m}$ is related, in the boson picture of nuclear excitation, to the excitation of $\mid 1_{i}^{-}>_{m}$ from the ground state as follows:

$$
<\left[1_{i^{\prime}}^{-} \otimes 1_{i}^{-}\right]_{J^{\pi}}\|E 1\| 1_{i}^{-}>=\sqrt{\left(1+\delta_{i, i^{\prime}}\right) \frac{(2 J+1)}{3}}<1_{i^{\prime}}^{-}\|E 1\| \text { g.s. }>.
$$

It should be noted that although for the two-phonon states, eq. (0.1), we have an extra factor $\sqrt{2}$, the states of eq. (0.2) play a more important role in two-step excitations since they can be reached by two different possibilities: g.s. $\rightarrow 1_{i}^{-} \rightarrow\left[1_{i}^{-} \otimes 1_{i^{\prime}}^{-}\right]$and g.s. $\rightarrow 1_{i^{\prime}}^{-} \rightarrow\left[1_{i}^{-} \otimes 1_{i^{\prime}}^{-}\right]$. Making use of these nuclear structure ingredients we have performed calculations of DGDR excitation in relativistic heavy ion collisions in second-order perturbation theory and with a coupled-channels procedure. 
The first effect we observed was that in second-order perturbation theory the amplitude for this process was identically zero in a semi-classical approach [17]. This can be understood by looking at figure 1 . The time-dependent field $V_{E 1}$ carries angular momentum with projections $m=0, \pm 1$. Thus, to reach the $1^{+}$DGDR magnetic substates, many routes are possible. The lines represent transitions caused by the different projections of $V_{E 1}$ : (a) dashed lines are for $m=0$, (b) dashed-dotted lines are for $m=-1$, and (c) solid-lines are for $m=+1$. The relation $V_{E 1, m=0} \neq V_{E 1, m= \pm 1}$ holds, so that not all routes yield the same excitation amplitude. Since the phases of the wavefunctions of each set of magnetic substates are equal, the difference between the transition amplitudes to a final $M$, can also arise from different values of the Clebsh-Gordan coefficients $\left(1 \mathrm{~m} 1 \mathrm{~m}^{\prime} \mid 1 \mathrm{M}\right)$. It is easy to see that, for any route to a final $M$, the second-order amplitude will be proportional to $(001 m \mid 1 m)\left(1 m 1 m^{\prime} \mid 1 M\right) V_{E 1, m^{\prime}} V_{E 1, m}+\left(m \leftrightarrow m^{\prime}\right)$. The two amplitudes carry opposite signs from the value of the Clebsh-Gordan coefficients. The identically zero result for the excitation amplitude of the $1^{+}$DGDR state is therefore a consequence of

$$
\sum_{m m^{\prime}}(001 m \mid 1 m)\left(1 m 1 m^{\prime} \mid 1 M\right)=0 .
$$

We have also performed a coupled-channels calculation following the theory described in ref. [18]. As shown in ref. [18], the coupling of the electric quadrupole (isovector and isoscalar) and the electric dipole states is very weak and can be neglected. We therefore include in our space only one-phonon $1^{-}$and two-phonon $\left[1_{i}^{-} \otimes 1_{i^{\prime}}^{-}\right]_{J^{\pi}}\left(J^{\pi}=0^{+}, 1^{+}\right.$and $\left.2^{+}\right)$ states. We obtain the occupation amplitudes by solving the coupled-channels equations

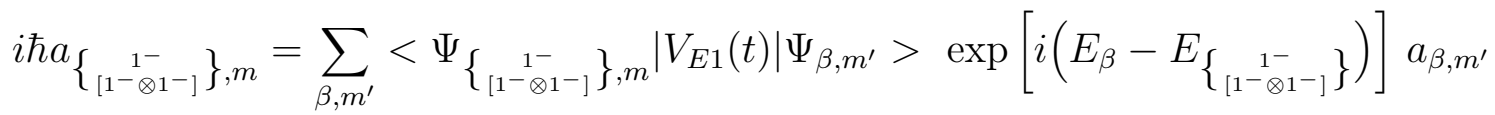

where $\beta=1_{i}^{-}$or $\left[1_{i}^{-} \otimes 1_{i^{\prime}}^{-}\right]$. The time dependent electric dipole field is that of a straight-line moving particle with charge $Z e$, and impact parameter $b$ (we use eqs. (25-26) of ref. [18]).

Due to the large number of degenerate magnetic substates, to make our coupled-channels calculation feasible, we have chosen a limited set of GDR and DGDR states. We have taken six $1^{-}$states which have the largest value of the reduced matrix element $<1_{i}^{-}\|E 1\|$ g.s. $>$. These six states exhaust $90.6 \%$ of the classical EWSR, while all $1^{-}$states up to $25 \mathrm{MeV}$ in our RPA calculation exhaust $94.3 \%$ of it. This value is somewhat smaller than the $122 \%$ reported in ref. [19]. It is because the continuum in our RPA calculation was approximated by narrow quasibound states. From these six one-phonon $1^{-}$states we construct two-phonon $\left[1_{i}^{-} \otimes 1_{i^{\prime}}^{-}\right]_{J^{\pi}}$ states, eqs. (0.1-0.2), which also have the largest matrix element of excitation 
$<\left[1_{i}^{-} \otimes 1_{i^{\prime}}^{-}\right]_{J^{\pi}}\|E 1\| 1_{i}^{-}>$for excitations starting from one-phonon states 円. The number of two-phonon states equals to twenty one for $J^{\pi}=0^{+}$and $2^{+}$, and to fifteen for $J^{\pi}=$ $1^{+}$. The cross section for the DGDR excitation was obtained by a sum over the final magnetic substates of the square of the occupation amplitudes and, finally, by an integration over impact parameter. We have chosen the minimum impact parameter, $b=15.54 \mathrm{fm}$, corresponding to the parameterization of ref. [21], appropriate for lead-lead collisions.

The electromagnetic excitation cross sections for the reaction ${ }^{208} \mathrm{~Pb}(640 \mathrm{MeV} /$ nucleon$)$ $+{ }^{208} \mathrm{~Pb}$ with excitation of all our basic 63 states is shown in figure 2. The total cross sections for each multipolarity are presented in table \, together with the results of first-order (for one-phonon excitations) and second-order (for two-phonon excitations) perturbation theory. The coupled-channels calculation yields a non-zero cross section for the $1^{+}$DGDR state due to other possible routes (higher-order), not included in second-order perturbation theory. One observes a considerable reduction of the DGDR cross sections, as compared to the predictions of the second-order perturbation theory. The GDR cross sections are also reduced in magnitude. However, the population of the $1^{+}$DGDR states are not appreciable and cannot be the source of the missing excitation cross section needed to explain the experiments. In general, the coupled-channels calculation practically does not change the relative contribution of different one-phonon $1_{i}^{-}$and two-phonon states $\left[1_{i}^{-} \otimes 1_{i^{\prime}}^{-}\right]_{J^{\pi}}$ to the total cross section with given $J^{\pi}=1^{-}, 0^{+}$and $2^{+}$. But since the $1^{+}$component of DGDR, with its zero value of excitation cross section in the second-order perturbation theory, has a special place among the two other components, the main effect of coupled-channels is to redistribute the total cross section between the $J^{\pi}=0^{+}, 2^{+}$and $J^{\pi}=1^{+}$components.

The calculated cross section in coupled-channels for both GDR and DGDR are somewhat smaller than reported in experimental findings [5]. This is not surprising since as mentioned above our chosen six $1^{-}$states exhaust only $90.6 \%$ of EWSR while the photo-neutron data

\footnotetext{
${ }^{1}$ It was demonstrated in ref. [16,20 that direct excitation of two-phonon configurations from the ground state is very weak. It allows us to exclude in our calculation matrix elements of the form $<\left[1_{i}^{-} \otimes 1_{i^{\prime}}^{-}\right]_{2^{+}\left(1^{+}\right)} \| E 2(M 1)|| g . s .>$ which correspond to direct transitions and produce higher order effects in comparison with accounted ones. These matrix elements give rise to DGDR excitation in first order perturbation theory. Thus, to prove our approximation we have calculated such cross sections and got total values equal to $0.11 \mathrm{mb}$ and ¡0.01 mb for the twenty one $2^{+}$and the fifteen $1^{+}$basic two-phonon states, respectively. These values have to be compared to $244.9 \mathrm{mb}$ for the total DGDR cross section in the second order perturbation theory.
} 
[19] indicate that this value equals to $122 \%$. Due to this underestimate of exhaust of the EWSR the cross section for DGDR excitation reduces more strongly than the one for the single GDR. This is because the GDR cross section is roughly proportional to the total $\mathrm{B}(\mathrm{E} 1)$ value while for DGDR it is proportional to the square of it. If we apply a primitive scaling to obtain the experimental value $122 \%$ of EWSR the ratio $R=\sigma_{(D G D R)} / \sigma_{(G D R)}$, the last line of our table [, changes into 0.096 and 0.101 for the coupled-channels calculation and for the perturbation theory, respectively. The experimental findings [5] yield the value $R_{\text {exp }}=0.116 \pm 0.014$. The reported [0] disagreement $R_{\text {exp }} / R_{\text {calc }}=1.33 \pm 0.16$ is the result of a comparison with $R_{\text {calc }}$ obtained within a folding model, assuming $122 \%$ of the EWSR. We get a somewhat larger value of $R_{\text {calc }}$ (taking into account our scaling procedure) because the $\mathrm{B}(\mathrm{E} 1)$ strength distribution over our six $1^{-}$states is not symmetrical with respect to the centroid energy, $E_{G D R}$ : the lower part is enhanced. A weak energy dependence in the excitation amplitude (see also ref. [8] for a discussion of this problem), which is also squared for the DGDR, enhances the DGDR cross section for a non-symmetrical distribution with respect to the symmetrical one, or when the GDR is treated as a single state. The effect of the energy dependence is demonstrated for a single GDR in the top part of figure 2 where the excitation cross sections are compared to the $\mathrm{B}(\mathrm{E} 1)$ strength distribution. It produces a shift to lower energies of the centroid of the GDR and the DGDR cross sections with respect to the centroid of the $\mathrm{B}(\mathrm{E} 1)$ and the $\mathrm{B}(\mathrm{E} 1) \times \mathrm{B}(\mathrm{E} 1)$ strength distribution, respectively. In our calculation this shift equals to $0.26 \mathrm{MeV}$ for the GDR and to $0.33,0.28 \mathrm{MeV}$ for the DGDR within coupled-channels and perturbation theory, respectively.

Of course, this scaling procedure has no deep physical meaning but we have included this discussion to indicate that the disagreement between experiment and theory for the DGDR excitation cross sections in ${ }^{208} \mathrm{~Pb}$ reached the stage when theoretical calculations have to provide a very precise description of both the GDR and the DGDR to draw up final conclusions. Work in this direction is in progress now and will be reported soon.

In summary, we have studied the excitation cross sections of the $1^{+}$component of the twophonon giant dipole resonance in ${ }^{208} \mathrm{~Pb}$ (together with $0^{+}$and $2^{+}$components) in relativistic heavy ion collisions. In second order perturbation theory this cross section equals to zero due to angular momentum properties. In coupled-channels calculations this component contributes to the total cross section. But it is appreciably quenched with respect to other components of DGDR. We indicate that a precise microscopic calculation of the giant dipole resonance is required to answer the question whether the problem of enhanced experimental 
cross section for DGDR excitation in relativistic heavy ion collisions stands for or not.

The authors thank H. Emling for fruitful discussions and suggestions and acknowledge financial support from the GSI facility at Darmstadt, where an essential part of this work has been done. C.A. B. acknowledges the Brazilian funding agencies CAPES, CNPq, and FUJB/UFRJ for partial support. V.Yu. P. and V.V. V. thank the RFBR (grant 95-02-05701) for partial support. 


\section{REFERENCES}

[1] R. Schmidt et al., Phys. Rev. Lett. 70 (1993) 1767

[2] T. Aumann et al., Phys. Rev.C47 (1993) 1728

[3] J.L. Ritmann et al., Phys. Rev. Lett. 70 (1993) 533 and 2659

[4] J.R. Beene et al., Nucl. Phys. A569 (1993) 163c

[5] J. Stroth et al., Nucl. Phys. 599 (1996) 307c;

K. Boretzky et al., GSI/1996 preprint, to be published

[6] H. Emling, Prog. Part. Phys. 33 (1994) 729;

Ph. Chomaz and N. Frascaria, Phys. Rep. 252 (1995) 275

[7] C.A. Bertulani and V.G. Zelevinsky, Phys. Rev. Lett. 71 (1993) 967; Nucl. Phys. A568 (1994) 931

[8] C. Volpe et al., A589 (1995) 521

[9] F. Catara, Ph. Chomaz and N. Van Giai, Phys. Lett. B233 (1989) 6

[10] V.Yu. Ponomarev et al., Phys. Rev. Lett. 72 (1994) 1168

[11] S. Nishizaki and J. Wambach, Phys. Lett. B349 (1995) 7

[12] V.Yu. Ponomarev et al., Nucl. Phys. 599 (1996) 341c

[13] V.Yu. Ponomarev, P.F. Bortignon, R.A. Broglia and V.V. Voronov, Milano/1996 preprint, to be published

[14] H. Kurasawa and T. Suzuki, Nucl. Phys. A597 (1996) 374

[15] V.G. Soloviev, Theory of Atomic Nuclei: Quasiparticles and Phonons (Institute of Physics, Bristol, 1992)

[16] V.Yu. Ponomarev and V.V. Voronov, Phys. Lett. B279 (1992) 1

[17] C.A. Bertulani and G. Baur, Phys. Rep. 163 (1988) 299

[18] C.A. Bertulani, L.F. Canto, M.S. Hussein and A.F.R. de Toledo Piza, Phys. Rev. C53 (1996) 334

[19] A. Vessiére et al., Nucl. Phys. A159 (1970) 561

[20] F. Catara, Ph. Chomaz and N. Van Giai, Phys. Lett. B277 (1992) 1 
[21] C.J. Benesh, J. Cook and J. Vary, Phys. Rev. C40 (1989) 1198 


\section{TABLES}

TABLE I. Cross section (in mb) for the excitation of the GDR and the three components with $J^{\pi}=0^{+}, 2^{+}, 1^{+}$of the DGDR in ${ }^{208} \mathrm{~Pb}(640 \mathrm{MeV} / \mathrm{A})+{ }^{208} \mathrm{~Pb}$ collisions. Calculations are performed within coupled-channels (CC) and within the first (PT-1) and second (PT-2) order perturbation theory, respectively.

\begin{tabular}{|l|r|r|c|}
\hline \hline \hline & $\mathrm{CC}$ & $\mathrm{PT}-1$ & $\mathrm{PT}-2$ \\
\hline$G D R$ & 2830 & 3275. & 0. \\
\hline$D G D R_{0^{+}}$ & 33.0 & 0. & 43.1 \\
$D G D R_{2^{+}}$ & 163.0 & 0.11 & 201.8 \\
$D G D R_{1^{+}}$ & 6.3 & $j 0.01$ & 0. \\
\hline \hline$D G D R / G D R$ & 0.071 & & 0.075 \\
\hline \hline
\end{tabular}




\section{FIGURES}

FIG. 1. The possible paths to the excitation of a given magnetic substate of the $1^{+}$component of the DGDR are displayed. The transitions caused by the different projections of the operator $V_{E 1}$ are shown by: (a) dashed lines for $m=0$, (b) dashed-dotted lines for $m=-1$, and (c) solid-lines are $m=+1$.

FIG. 2. The electromagnetic excitation cross sections for the reaction ${ }^{208} \mathrm{~Pb}(640 \mathrm{MeV} /$ nucleon$)$ $+{ }^{208} \mathrm{~Pb}$ calculated in coupled-channels. It is shown the excitation of the GDR (top) and the three

components $J^{\pi}$ arbitrary units) over $1^{-}$states is shown by dashed lines. For visuality it is shifted up by $100 \mathrm{keV}$. 
This figure "fig1-1.png" is available in "png" format from: http://arxiv.org/ps/nucl-th/9606004v1 
This figure "fig2-1.png" is available in "png" format from: http://arxiv.org/ps/nucl-th/9606004v1 